\title{
Progesterone: Examination of its Postulated Inhibitory Actions on Lordosis During the Rat Estrous Cycle'
}

\author{
J. BRADLEY POWERS AND JUDITH MOREINES \\ Neuroscience Laboratory and Department of Psychology, 1103 E. Huron, University of Michigan \\ Ann Arbor, MI 48109
}

(Received 20 October 1976)

\begin{abstract}
POWERS, J. B. AND J. MOREINES. Progesterone: examination of its postulated inhibitory actions on lordosis during the rat estrous cycle. PHYSIOL. BEHAV. 17(3) 493-498, 1976. - Three experiments tested whether the inhibitory effects of progesterone could be of physiological significance in regulating the duration of behavioral estrus in female rats. In animals displaying 5 day estrous cycles, a second period of sexual receptivity, one day following the occurrence of spontaneous estrus, could be induced by exogenous hormone administration, regardless of whether the ovaries were intact or were removed during the period over which the exogenous hormones were acting. In a second experiment, acute ovariectomy at various times during the progesterone surge acted only to degrade the quality of receptive behavior subsequently observed, never to enhance it by removing a postulated inhibitory influence. In the final experiment there was some suggestion that progesterone's facilitating effect on lordosis during the later portions of spontaneous estrus were attenuated by prior exposure to ovarian secretions during the early period of behavioral estrus. Our results are consistent with the hypothesis that the duration of receptive behavior under physiological conditions is not primarily regulated by inhibitory actions of progesterone, but rather by the quantity and duration of estrogen secretions during the conditioning period.
\end{abstract}

Progesterone Biphasic effects Lordosis Estrous cycle Estrogen Acute ovariectomy

SEXUAL receptivity of many female rodents is regulated by the sequential actions of estrogen and progesterone $[9,16]$. It is currently thought that estrogen in some way primes critical target cells in the central nervous system and that progesterone subsequently facilitates the onset of lordosis responding by interacting with this neural substrate. Although the hormonal events associated with the onset of estrus have been elucidated $[3,11,22]$, factors controlling the length of time that a female remains sexually receptive are less well understood. It has been proposed that the inhibitory effects of progesterone constitute one of these factors [15]. According to this view, the influence of progesterone is biphasic; it initially facilitates the onset of lordosis but later becomes inhibitory, and in this way terminates the period of sexual receptivity.

There is some evidence for biphasic progesterone effects in guinea pigs, hamsters, mice and rats $[4,5,7,19,21,25$, 27,301 . However, it is important in evaluating these studies to distinguish between two types of inhibitory actions which progesterone might exert on estrogen-dependent behavior. We shall refer to these two types as concurrent and sequential. Concurrent inhibition can occur when progesterone is present during the time of estrogen priming. An example of this is provided by the demonstration that progesterone injections, either prior to, concurrent with, or shortly after estrogen administration, can attenuate the effect this estrogen treatment would normally have on lordosis $[5,25,27]$. Sequential progesterone inhibition, on the other hand, occurs when the inhibitory action is exerted subsequent to the completion of estrogen conditioning. This has been demonstrated by examining the effects of progesterone injections over two successive days in estrogen-primed females $[4,7,19,21,30]$. The first progesterone treatment facilitates sexual responsiveness, but also in some way renders the nervous system refractory to the second progesterone injection.

Most investigations of biphasic progesterone effects have not distinguished between these two types of inhibition. If inhibitory effects of progesterone have functional significance for the regulation of rodent estrous cycles, they should be of the sequential type. This is because under physiological conditions there are minimal quantities of progesterone in the systemic circulation during the time that estrogen levels are rising and estrogen priming of lordosis is taking place. The progesterone surge which facilitates lordosis begins at approximately the same time that estrogen levels start to decline from their preovulatory peak $[2,10,13,24]$. It is not likely then that progesterone inhibition of receptive behavior would be mediated pri-

\footnotetext{
${ }^{1}$ Research supported by NIH Grant No. 1 R01 HD08281-01.
} 
marily by a suppression of estrogen-conditioning processes.

This report focuses on the possible sequential inhibitory effects of progesterone in the rat. There are two studies which demonstrate that a biphasic progesterone inhibition of lordosis can occur in chronically ovariectomized rats $[14,21]$. A later report, however, indicated that such an effect was not necessarily of significance for regulating the duration of behavioral estrus under physiological conditions [22]. Barfield and Lisk [1] have recently challenged this conclusion so the question remains whether progesterone in part regulates the termination of behavioral estrus in rats. It is important to point out that in the latter studies using females displaying regular estrous cycles $[1,22]$, the possible inhibitory effects of endogenous progesterone examined were of the concurrent, not the sequential type. In both cases, the display of lordosis one day after its expected time of occurrence during the estrous cycle was studied. In order to induce this additional period of sexual receptivity, supplementary estrogen and progesterone must be provided. If the supplementary estrogen is injected at a time when endogenous progesterone levels are high, this progesterone may act to attenuate subsequent receptive behavior primarily by blocking the ability of estrogen to reestablish an appropriately conditioned neural substrate. This is an example of concurrent inhibition.

The experiments presented here reexamined this action of progesterone but focused more directly on the possible sequential effects of this hormone during the rat estrous cycle.

\section{GENERAL PROCEDURES}

Sprague-Dawley rats (Holtzman; Madison, Wisconsin) were housed individually under a $14 \mathrm{hr}$ light: $10 \mathrm{hr}$ dark illumination schedule with lights on from 2100 until 1100 . Each day during the dark portion of the photoperiod, all females were manually stimulated in the flank and genital regions in order to detect the presence or absence of the lordosis response. This technique permits a simple and reliable monitoring of estrous cycle length [29]. A minimum of 2 consecutive 5 day cycles were observed before any female was designated for experimental use.

Surgical procedures, steroid injections, and behavioral tests were conducted at various times with respect to a reference point in the estrous cycle. This point was designated $0 \mathrm{hr}$ and coincided with the beginning of the critical period for luteinizing hormone release. With the light cycle used in the present experiments, this time corresponded to 0600 . Figure 1 depicts $0 \mathrm{hr}$ and other relevant experimental times superimposed upon a schematic representation of the temporal patterns of estradiol and progesterone secretion during the 5 day estrous cycle. Although the exact quantities of these hormones, expressed either as blood levels or ovarian secretion rates, vary according to the reporting laboratory and the measurement techniques used, the gradual rise in estradiol over a $1-2$ day period prior to $0 \mathrm{hr}$, and a pronounced increase in progesterone levels subsequent to $0 \mathrm{hr}$, are consistently found $[2,10,13,24]$. The importance of the arbitrary time scale used for the present experiments is that the hormonal secretions necessary for estrogen conditioning occur prior to $0 \mathrm{hr}$ whereas the progesterone surge which is necessary to facilitate the onset of lordosis occurs after 0 hr. Ovariectomies (OVX) or sham ovariectomies (SOVX) were performed under methoxyflurane anesthesia (Pen-

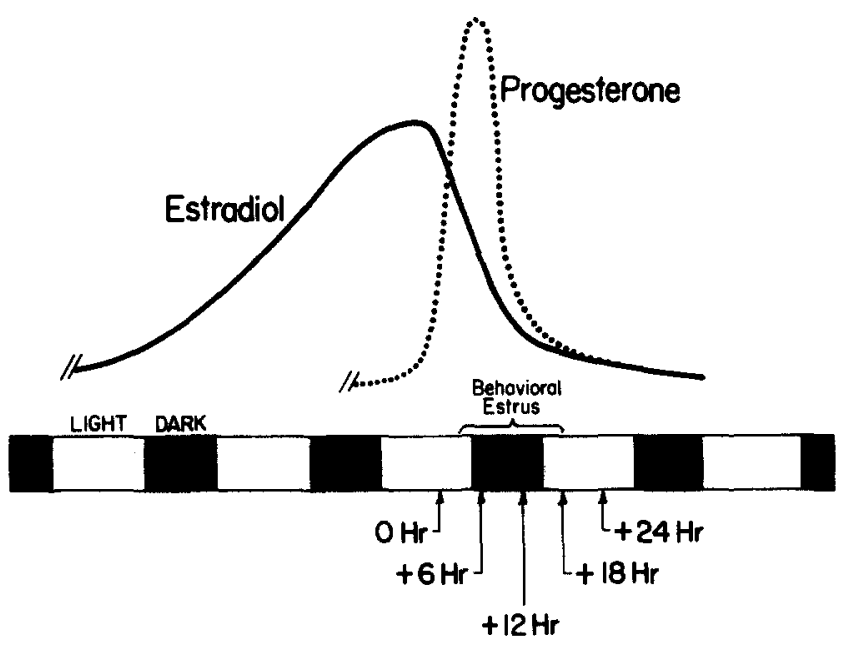

FIG. 1. Schematic depiction of estradiol and progesterone secretory patterns during 5 day estrous cycles with times indicated when experimental manipualtions were performed. The arbitrary origin $(0$ hr) corresponds with the onset of the critical period for luteinizing hormone release and precedes the time during which behavioral estrus is maximally displayed.

thrane, Abbott Laboratories) using a single ventral midline incision. For SOVX, the abdominal musculature was cut and the uterine horns visualized, but the ovaries were not manipulated; for OVX the uterine horns were ligated and the ovaries removed. When required, steroids were injected subcutaneously (SC) in sesame oil.

Behavior tests to determine the intensity of sexual receptivity were performed by scoring the lordosis responses elicited by sexually active Long-Evans male rats previously habituated to semicircular mating arenas $(76 \mathrm{~cm}$ dia. and $40 \mathrm{~cm}$ in height and width). The quality of each lordosis response following vigorous mounting attempts was rated on a scale from $0-3: 0=$ no concave arching of the back; 1,2 and 3 = slight, moderate, and full lordosis, respectively. Responses to 10 adequate male mounts were obtained and the mean response value was used as the receptivity score (RS). RS differences between groups were assessed by the Mann-Whitney or Wilcoxon tests, where appropriate.

\section{EXPERIMENT 1}

Female rats become sexually receptive for approximately $15 \mathrm{hr}$ during spontaneous behavioral estrus [16]. This occurs on the average between $+3 \mathrm{hr}$ and $+18 \mathrm{hr}$ (Fig. 1), that is, concurrently with the increase in blood levels of progesterone [8]. A second period of receptive behavior can be induced on the following day by administering supplementary estrogen at $0 \mathrm{hr}$ and progesterone 24 to 30 hr later $[1,22]$. However, this effect can apparently be modified by endogenous ovarian secretions. If females are ovariectomized at $0 \mathrm{hr}$, thus eliminating the progesterone surge, this second behavioral estrus can be induced; if the ovaries are not removed until $+24 \mathrm{hr}$, the exogenous estrogen and progesterone treatment appears quite ineffective [1]. Previous work from our laboratory had indicated that if the ovaries were removed at $+7 \mathrm{hr}$, sufficient progesterone had been secreted by that time to facilitate receptive behavior when tested a few hr later; however, exposure to this quantity and duration of 
progesterone was not sufficient to inhibit a second period of behavioral estrus the next day. It appears then that the endogenous progesterone surge must be left intact for longer than $7 \mathrm{hr}$ if these inhibitory effects are to become manifest. In Experiment 1 we tested this suggestion by examining the concurrent inhibitory effects of progesterone secreted between $0 \mathrm{hr}$ and $+24 \mathrm{hr}$ (Fig. 1).

\section{Method}

In our colony only a small percentage of females exhibit 4 day estrous cycles; consequently we restricted our investigations to females with cycles of 5 days duration. After displaying two such consecutive cycles each animal was assigned to 1 of 2 groups. On the day of the next expected behavioral estrus, $\mathrm{Gr} 1(\mathrm{n}=6)$ was $\mathrm{OVX}$ at $0 \mathrm{hr}$, while Gr $2(n=8)$ was OVX 1 day later at $+24 \mathrm{hr}$. The expected days of behavioral estrus were confirmed either by an accumulation of intraluminal fluid in the uterine horns at the time of ovariectomy in $\mathrm{Gr} 1(0 \mathrm{hr})$, or by the presence of lordosis during the daily manual stimulation tests in $\mathrm{Gr} 2(+9 \mathrm{hr})$. In the latter case, females still had intact ovaries and consequently should have displayed typical receptive behavior on the predicted day of behavioral estrus. Both $\mathrm{Gr} 1$ and 2 received estradiol at $0 \mathrm{hr}$ $(26.7 \mu \mathrm{g} / \mathrm{kg}$ b.w.), progesterone at $+27 \mathrm{hr}(500 \mu \mathrm{g} /$ animal $)$, and behavior tests at $+33 \mathrm{hr}$. Thus the behavioral effects of exogenous estrogen and progesterone were assessed in females whose ovaries were either removed ( $\mathrm{Gr} 1)$ or left in situ (Gr 2) during the period of endogenous progesterone release.

\section{Results}

The intensity of sexual receptivity was essentially identical in Gr 1 and Gr 2 females. (The mean and standard error of their receptivity scores were $2.15 \pm 0.36$ and $2.16 \pm$ 0.20 , respectively.) The presence or absence of ovarian secretions between $0 \mathrm{hr}$ and $+24 \mathrm{hr}$ did not affect the intensity of lordosis behavior induced by exogenous hormone injections. The mean RS of both groups was slightly less than the receptivity which would be displayed by females in natural estrus (RS $=2.8-3.0$, unpublished); however, this difference was due mostly to the quantity of estradiol injected at $0 \mathrm{hr}$.

\section{EXPERIMENT 2}

Although Experiment 1 failed to demonstrate a progesterone antagonism of sexual receptivity, the procedures we used were not identical to those of Barfield and Lisk [1]. In both cases, however, the paradigms employed were not optimal for assessing inhibitory effects of progesterone which might be functionally significant during the estrous cycle. Experiment 2 was an attempt to test more directly the possible sequential inhibitory effects of progesterone. According to one view of these sequential actions, the behavioral effects of progesterone secreted concomitantly with the onset, maintenance, and termination of sexual receptivity are mediated by 2 distinct components. After appropriate estrogen conditioning has taken place, the first component facilitates sexual responsiveness. Females not exposed to this progesterone action should be less receptive than females who have been so exposed. The second component is inhibitory, causing opposite functional effects; it actively suppresses lordosis response mechanisms.
Consequently, if this second component is prevented from acting, females should be more receptive or remain receptive for a longer period of time than should females which have been exposed to both the facilitatory and inhibitory actions of progesterone. These predictions were tested in Experiment 2 by assessing the behavioral effects of ovariectomy in different groups of females at varying times following $0 \mathrm{hr}$.

\section{Method}

Females displaying 5 day cycles were OVX or SOVX at either $+6 \mathrm{hr}(\mathrm{Gr} \mathrm{3}),+12 \mathrm{hr}(\mathrm{Gr} \mathrm{4})$, or $+18 \mathrm{hr}(\mathrm{Gr} \mathrm{5})$ and were tested for sexual receptivity $6 \mathrm{hr}$ after surgery. $\mathrm{Gr}$ 3-OVX and Gr 3-SOVX had 20 and 21 females, respectively; there were 5 animals in each of the remaining 4 conditions. If the effects of ovarian progesterone shift from facilitation to inhibition at some point between +6 and +24 $\mathrm{hr}$, females undergoing OVX at one or more of these experimental times should display higher receptivity scores than sham-operated controls because their exposure to the inhibitory actions of progesterone would have been decreased.

\section{Results}

Females OVX at either $+6,+12$, or $+18 \mathrm{hr}$, had lower receptivity scores than their SOVX controls (Fig. 2), but these differences were significant only for $\mathrm{Gr} 3$ females $(p<0.05)$. In none of the groups did OVX females display better lordosis responding than did their sham-operated controls. These findings are consistent with the idea that ovarian secretions between +6 and $+18 \mathrm{hr}$ facilitate rather than inhibit lordosis. Because sexual responsiveness was minimal in both OVX and SOVX groups following $+18 \mathrm{hr}$ surgery, ovarian secretions after this time appear to contribute little to the control of lordosis in 5 day estrous cycles.

\section{EXPERIMENT 3}

The results of Experiment 2 do not support the hypothesis that progesterone regulates the sexual receptivity of female rats by acting biphasically during the

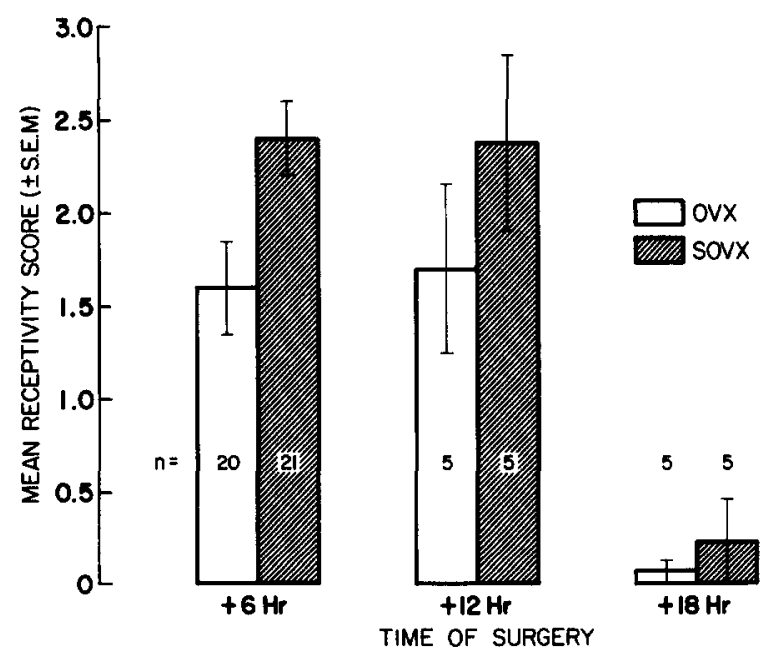

FIG. 2. Receptive behavior $6 \mathrm{hr}$ following ovariectomy or sham ovariectomy performed at various times during behavioral estrus. 
estrous cycle. However, there are alternative means by which progesterone could inhibit receptive behavior under physiological conditions. Rather than actively suppressing lordosis response mechanisms, progesterone might modulate the sensitivity of relevant neural substrates to its own facilitatory effects. More specifically, this variant of the sequential inhibition hypothesis suggests that the efficacy by which progesterone can synergize with estrogen to promote lordosis during the late stages of behavioral estrus is attenuated by the facilitatory actions of progesterone which occur during early estrus. To test this idea, rats were ovariectomized during this early period of behavioral estrus, and tested subsequently for responsiveness to exogenous progesterone administration. According to the hypothesis, elimination of some portion of the early facilitatory actions of progesterone should have 2 major effects: (1) sexual responsiveness should be lower after OVX than after SOVX in the absence of any other treatment (Exp. 2); and (2) following subsequent exogenous progesterone treatment, receptivity scores should be higher in OVX than in SOVX females. This is because removing progesterone prevents it from desensitizing the brain to its own lordosis-facilitating effects. Thus females exposed to this desensitization (SOVX) should respond less well to subsequent progesterone treatment than should females in which this inhibitory action has been eliminated (OVX).

\section{Method}

Females exhibiting 5 day estrous cycles were used in 1 of 2 groups ( $\mathrm{Gr} 6-7$ ). In each group, various treatments and tests were administered sequentially on the day of expected behavioral estrus at $+6 \mathrm{hr},+12 \mathrm{hr}$, and $+18 \mathrm{hr}$. Within each group, approximately half the animals were assigned to an $\mathrm{o}-\mathrm{s}$ condition and the remaining animals to an s-o condition. Condition o-s involved OVX at $+6 \mathrm{hr}$ and SOVX at +12 hr while in condition s-o, the order of these 2 surgical procedures was reversed. Gr 6 females were tested for sexual receptivity just prior to the $+12 \mathrm{hr}$ surgery, injected with $100 \mu \mathrm{g} \mathrm{P}$ immediately after the OVX or SOVX, and given a final behavior test at $+18 \mathrm{hr}$. Gr 7 females were treated comparably except that the dose of exogenous $\mathrm{P}$ was increased to $500 \mu \mathrm{g} / \mathrm{animal}$. Thus for each of the groups, the behavioral effects of exogenous progesterone given at $+12 \mathrm{hr}$ were tested $6 \mathrm{hr}$ later as a function of the absence or presence of ovarian secretions between +6 and +12 hrs (conditions o-s and s-o, re- spectively). The sequence of treatments for all 4 experimental conditions is shown in Table 1.

\section{Results}

The presence of ovarian secretions between +6 and +12 hrs modified subsequent responsiveness to injected progesterone but the absolute magnitude of this effect was not robust.

In both conditions of $\mathrm{Gr} 6,+18 \mathrm{hr}$ response scores were significantly lower than those observed on the $+12 \mathrm{hr}$ test ( $p<0.01$, Wilcoxon) but the $+18 \mathrm{hr}$ responsiveness was not different between the 2 conditions. Thus exogenous progesterone at $+12 \mathrm{hr}$ failed to maintain sexual receptivity, and this failure was equally evident regardless of whether ovarian secretions were present or absent during the $6 \mathrm{hr}$ period preceding the progesterone injection. These results suggested that the quantity of progesterone injected (100 $\mu \mathrm{g} /$ animal) was perhaps too low to be sensitive to the effects of OVX at $+6 \mathrm{hr}$. For this reason, the dose was increased to $500 \mu \mathrm{g} /$ animal for the females in $\mathrm{Gr} 7$. Under these conditions, the facilitating actions of exogenous progesterone treatment were affected by OVX at $+6 \mathrm{hr}$. Females in $\mathrm{Gr} 7$ o-s displayed a sexual responsiveness at +18 hr which was higher, but not significantly so, than their responsiveness at $+12 \mathrm{hr}$. This was not the case in $\mathrm{Gr} 7 \mathrm{~s}-\mathrm{O}$ animals in which $+18 \mathrm{hr}$ RS's were significantly lower than the $+12 \mathrm{hr}$ scores $(p<0.01)$. However, responsiveness at +18 hr was not significantly different between the two conditions of $\mathrm{Gr} 7$. Thus, when ovarian secretions were present up to $+12 \mathrm{hr}$, subsequent treatment with $500 \mu \mathrm{g}$ progesterone failed to sustain sexual receptivity over the next 6 hr ( $\mathrm{Gr} 7 \mathrm{~s}-\mathrm{o}$ ), whereas removal of the ovaries at $+6 \mathrm{hr}$ allowed the same quantity of progesterone at $+12 \mathrm{hr}$ to actually improve behavioral responsiveness $6 \mathrm{hr}$ later $(\mathrm{Gr} 7$ o-s). These findings are consistent with the hypothesis that endogenous ovarian secretions prior to $+12 \mathrm{hr}$ not only facilitate lordosis but in some manner attenuate the sensitivity of relevant neural mechanisms to the subsequent facilitatory actions of progesterone.

\section{DISCUSSION}

The findings reported here are consistent with the hypothesis that in rats, ovarian progesterone regulates behavioral estrus primarily by synergizing with estrogen to facilitate lordosis (Fig. 2). This conclusion is consistent

TABLE 1

EFFECTS OF EXOGENOUS PROGESTERONE TREATMENT FOLLOWING VARIOUS OVARIAN MANIPULATIONS DURING SPONTANEOUS BEHAVIORAL ESTRUS

\begin{tabular}{|c|c|c|c|c|c|c|}
\hline Group & $\mathbf{n}$ & $\begin{array}{l}+6 \mathrm{Hr} \\
\text { Surg* }\end{array}$ & RS $†$ & $\begin{array}{c}+12 \mathrm{Hr} \\
\text { Surg }\end{array}$ & Prog $\ddagger$ & $\begin{array}{c}+18 \mathrm{Hr} \\
\mathrm{RS}\end{array}$ \\
\hline $60-s$ & 10 & ovx & $1.83 \pm .40$ & SovX & 100 & $0.34 \pm 18 \S$ \\
\hline $6 s-0$ & 11 & SOVX & $2.34 \pm .29$ & ovX & 100 & $1.1 \pm .33 \S$ \\
\hline 7 o-s & 10 & ovX & $1.41 \pm .34$ & SOVX & 500 & $1.84 \pm .22$ \\
\hline $7 \mathrm{~s}-0$ & 10 & Sovx & $2.44 \pm .27$ & ovx & 500 & $1.08 \pm .368$ \\
\hline
\end{tabular}

*Surg-Ovariectomy (OVX) or Sham Ovariectomy (SOVX).

†RS-Mean Receptivity Score \pm SEM.

$\ddagger$ Prog-Progesterone ( $\mu \mathrm{g} / \mathrm{rat})$.

$\S \mathrm{RS}$ at $+12 \mathrm{hr}$ and $+18 \mathrm{hr}$ different- $p<0.01$, Wilcoxon test. 
with earlier findings from our laboratory [22 \] but should be tempered by the present results showing that ovarian secretions associated with the initial facilitation of lordosis also in some way reduce the subsequent effectiveness of progesterone (Experiment 3).

In Experiment 1 we were unable to replicate the inhibitory effects of ovarian secretions reported by Barfield and Lisk [1]. A second period of receptivity following spontaneous behavioral estrus could be induced by exogenous estradiol and progesterone treatment regardless of whether the ovaries were removed at $0 \mathrm{hr}$, thus eliminating the endogenous release of progesterone, or were left in situ until $+24 \mathrm{hr}$, in which case endogenous progesterone was secreted for an additional day. We must consider that these discrepant findings may in part be due to different experimental procedures used in the two laboratories. First, our females exhibited 5 day rather than 4 day estrous cycles; secondly we did not test for receptivity at $+24 \mathrm{hr}$ just prior to exogenous progesterone treatment; and thirdly the dose of progesterone we injected was considerably less $(500 \mu \mathrm{g} /$ animal vs $6700 \mu \mathrm{g} / \mathrm{kg} \mathrm{b} . \mathrm{w}$.$) .$

Although each of these factors may have contributed to our differing results, it is perhaps most important to emphasize that the necessity of administering exogenous estradiol in this general paradigm limits its usefulness for studying the physiological regulation of sexual receptivity. Because of this, the possibility that progesterone might exert sequential, rather than concurrent inhibitory effects in regulating the duration of behavioral estrus was tested in Experiments 2 and 3. In Experiment 2, we tested the hypothesis that removing an ovarian source of inhibition at times when females were displaying spontaneous behavioral estrus would result in enhanced or prolonged receptivity scores subsequent to ovariectomy. This hypothesis was not confirmed; females ovariectomized at either $+6,+12$, or $+18 \mathrm{hrs}$ were less receptive $6 \mathrm{hr}$ later than were females sham ovariectomized at comparable times (Fig. 2). Although the difference between OVX and SOVX receptivity scores was substantial in both the $+6 \mathrm{hr}$ and $+12 \mathrm{hr}$ groups, significance was achieved only in the former which may have been partly due to the greater number of animals in these groups. Thus at none of the 3 times during the period of endogenous progesterone release did removal of this hormone stimulus diminish an inhibitory influence on receptive behavior; on the contrary ovarian secretions during this period appear primarily to facilitate the lordosis response.

In Experiment 3, an alternative way in which endogenous gonadal hormones might exert inhibitory actions was tested. Specifically, we asked whether the activation of mechanisms by which progesterone facilitates sexual receptivity might reduce the hormonal sensitivity of these same mechanisms later during the estrus period. Our results (Table 1) indicate that this may be true although the absolute magnitude of this effect is not large. In Gr 6 females, receptivity levels were significantly lower at $+18 \mathrm{hr}$ than they were at $+12 \mathrm{hr}$. Because this was true for both the $\mathrm{o}-\mathrm{s}$ and $\mathrm{s}-\mathrm{o}$ conditions, we considered the possibility that $100 \mu \mathrm{g}$ progesterone was not an adequate replacement dose. That this might be the case was also suggested by the fact that in Experiment 2, females SOVX at $+12 \mathrm{hr}$ and tested at $+18 \mathrm{hr}$ (hence retaining their ovaries up to the time of testing) had a mean RS of 2.4. This contrasts with the $+18 \mathrm{hr}$ scores of $\mathrm{Gr} 6 \mathrm{~s}-\mathrm{o}$ females (mean $\mathrm{RS}=1.1$ ), suggesting that the $100 \mu \mathrm{g}$ progesterone supplement at +12 hr was not equivalent to what the ovaries normally secrete between +12 and $+18 \mathrm{hr}$. For these reasons, Gr 7 females were administered $500 \mu \mathrm{g}$ progesterone. Under these conditions exogenous progesterone treatment at $+12 \mathrm{hr}$ failed to maintain receptivity scores at their preinjection levels (measured at $+12 \mathrm{hr}$ ) if the ovaries remained in situ until $+12 \mathrm{hr}(\mathrm{Gr} 7 \mathrm{~s}-\mathrm{o})$. When ovariectomy was performed at $+6 \mathrm{hr}$ ( $\mathrm{Gr} 7 \mathrm{o}-\mathrm{s})$, thus eliminating the postulated inhibitory influence of endogenous ovarian secretions, exogenous progesterone at $+12 \mathrm{hr}$ was able to counteract the decline in receptivity scores shown by $\mathrm{Gr} 7$ s-o females. These results support the hypothesis that the effectiveness by which progesterone can facilitate lordosis is attenuated by prior progesterone exposure. Although we have no independent evidence that the relevant hormonal stimulus removed by OVX at $+6 \mathrm{hr}$ was progesterone, this conclusion is supported by the fact that progesterone is the major steroid secreted at this time with effects on lordosis $[26,28]$, and that exogenous progesterone injections in chronically ovariectomized rats can exert sequential inhibitory effects under appropriate conditions [21]. It should also be emphasized that there is no necessary reason why progesterone's initial effect of facilitating estrus need be a precondition for the manifestation of subsequent inhibitory actions. In ovariectomized guinea pigs, sequential inhibition has been demonstrated in the absence of prior facilitation [19] and likewise facilitation can occur without subsequent inhibition [30]. In this species the independence of these dual progesterone effects can be attributed in part to differential sites of action within the brain $[18,20]$. Thus in Experiment 3 it is conceivable that the endogenous secretion of progesterone could have diminished the effectiveness of exogenous progesterone injected at +12 hr by mechanisms quite independent of those responsible for the facilitation of lordosis.

Earlier in this paper we emphasized a distinction between concurrent and sequential inhibitory effects of progesterone. This was done to focus more clearly on hormonal mechanisms with physiological significance during the estrous cycle. It is now clear the sequential inhibitory actions of progesterone may play some role in limiting the time over which female rats remain sexually receptive during spontaneous behavioral estrus. It seems apparent, though, this is only one of many factors which regulate estrus duration, and that it probably is of limited significance. The quantity and duration of estradiol secretion prior to $0 \mathrm{hr}$ may be the most important variables in determining how long a female remains sexually responsive, assuming that adequate progesterone for facilitation is available $[6,23]$. Secondly, the actual time course of progesterone secretion following $0 \mathrm{hr}$ dictates the availability of this facilitatory stimulus. The fact that exogenous estrogen, in addition to that provided by the ovaries, is needed to extend behavioral estrus beyond its normal duration emphasizes the critical role of endogenous estradiol in setting limits on estrous duration ([1, 3, 22]; Experiment 1). The possibility that declining progesterone levels occuring late in estrus contribute importantly to diminished sexual responsiveness is not likely because of our earlier work indicating that supplementary progesterone, given in an attempt to prolong spontaneous behavioral estrus, is quite ineffective, regardless of whether the endogenous progesterone surge had been allowed to act or had been prevented by OVX at $0 \mathrm{hr}$ [22]. Because these artificially maintained progesterone levels do not extend 
the normal period of sexual receptivity, it is obvious that a decline in circulating progesterone is not a necessary condition for the termination of estrus $[12,17]$.

Inhibitory effects of progesterone on lordosis have been demonstrated using a variety of species and with different experimental paradigms. However, even for guinea pigs and hamsters where these effects appear pronounced, there is no unequivocal evidence that progesterone is of crucial significance for terminating receptive behavior during the estrous cycle. One problem in such an analysis is the impossibility of testing the independent effects of endogenously secreted estradiol and progesterone on estrus duration. This is because during behavioral estrus occurring physiologically, not only is progesterone being secreted which facilitates lordosis, but estradiol is also continuing to be released, even though blood levels are declining (Fig. 1). We have preliminary data to suggest, however, that the effects of this continued estradiol secretion on the duration of behavioral estrus are not being impeded or inhibited by the concurrent presence of progesterone.

\section{REFERENCES}

1. Barfield, M. A. and R. D. Lisk. Relative contributions of ovarian and adrenal progesterone to the timing of heat in the 4-day cyclic rat. Endocrinology 94: 571-575, 1974.

2. Butcher, R. L., W. E. Collins and N. W. Fugo. Plasma concentration of $\mathrm{LH}, \mathrm{FSH}$, prolactin, progesterone and estradiol-17B throughout the 4-day estrous cycle of the rat. Endocrinology 94: 1704-1708, 1974.

3. Ciaccio, L. A. and R. D. Lisk. Hormonal control of cyclic estrus in the female hamster. Am. J. Physiol. 221: 936-942, 1971.

4. Ciaccio, L. A. and R. D. Lisk. The role of progesterone in regulating the period of sexual receptivity in the female hamster. J. Endocr. 50: 201-207, 1971.

5. Czaja, J. A., D. A. Goldfoot and H. J. Karavolas. Comparative facilitation and inhibition of lordosis in the guinea pig with progesterone, 5a-pregnane-3,20-dione, or 3a-hydroxy-5apregnan-20-one. Hormones Behav. 5: 261-274, 1974.

6. Eaton, G. G., R. W. Goy and J. A. Resko. Brain uptake and metabolism of estradiol benzoate and estrous behavior in ovariectomized guinea pigs. Hormones Behav. 6: 81-97, 1975.

7. Edwards, D. A. Induction of estrus in female mice: EstrogenProgesterone interactions. Hormones Behav. 1: 299-304, 1970.

8. Feder, H. H., J. A. Resko and R. W. Goy. Progesterone levels in the arterial plasma of pre-ovulatory and ovariectomized rats. $J$. Endocr. 41: 563-569, 1968.

9. Gorski, R. A The neuroendocrine regulation of sexual behavior. In: Advances in Psychobiology, vol. 2, edited by G. Newton and A. M. Riesen. New York: Wiley, 1974, pp. 1-58.

10. Horikoshi, H. and Y. Suzuki. On circulating sex steroids during the estrous cycle and the early pseudopregnancy in the rat with special reference to its luteal activation. Endocr. Japon. 21: $69-79,1974$.

11. Josyln, W. D., H. H. Feder and R. W. Goy. Estrogen conditioning and progesterone facilitation of lordosis in guinea pigs. Physiol. Behav. 7: 477-482, 1971.

12. Joslyn, W. D., K. Wallen and R. W. Goy. Cyclic changes in sexual response to exogenous progesterone in female guinea pigs. Physiol. Behav. 7:915-917, 1971.

13. Kalra, S. P. and P. S. Kalra. Temporal interrelationships among circulating levels of estradiol, progesterone and LH during the rat estrous cycle: Effects of exogenous progesterone. Endocrinology 95: $1711-1718,1974$.

14. Lisk, R. D. Progesterone: Biphasic effects on the lordosis response in adult or neonatally gonadectomized rats. Neuroendocrinology 5: 149-160, 1969.

15. Lisk, R. D. Progesterone: Role in limitation of ovulation and sex behavior in mammals. Trans. N. Y. Acad. Sci. 31: $593-601,1969$.
16. Lisk, R. D. Hormonal regulation of sexual behavior in polyestrous mammals common to the laboratory. In: Handbook of Physiology - Endocrinology II, Part 1, edited by R. O. Greep. American Physiological Society, Bethesda, 1973, pp. 223-260.

17. Morin, L. P. and H. H. Feder. Multiple progesterone injections and the duration of estrus in ovariectomized guinea pigs. Physiol. Behav. 11: 861-865, 1973.

18. Morin, L. P. and H. H. Feder. Hypothalamic progesterone implants and facilitation of lordosis behavior in estrogenprimed ovariectomized guinea pigs. Brain Res. 70: 81-93, 1974.

19. Morin, L. P. and H. H. Feder. Independence of progesteroneinduced facilitation and inhibition of lordosis behavior in ovariectomized guinea pigs. Hormones Behav. 5: 7-12, 1974.

20. Morin, L. P. and H. H. Feder. Inhibition of lordosis behavior in ovariectomized guinea pigs by mesencephalic implants of progesterone. Brain Res. 70: 71-80, 1974.

21. Nadler, R. D. A biphasic influence of progesterone on sexual receptivity of spayed female rats. Physiol. Behav. 5: 95-97, 1970.

22. Powers, J. B. Hormonal control of sexual receptivity during the estrous cycle of the rat. Phsyiol. Behav. 5: 831-835, 1970.

23. Quadagno, D. M., J. McCullough and R. Langan. The effect of varying amounts of exogenous estradiol benzoate on estrous behavior in the rat. Hormones Behav. 3: 175-179, 1972.

24. Smith, M. S., M. E. Freeman and J. D. Neill. The control of progesterone secretion during the estrous cycle and early pseudopregnancy in the rat: Prolactin, gonadotropin and steroid levels associated with rescue of the corpus luteum of pseudopregnancy. Endocrinology 96: 219-226, 1975.

25. Wallen, K., R. W. Goy and C. H. Phoenix. Inhibitory actions of progesterone on hormonal induction of estrus in female guinea pigs. Hormones Behav. 6: 127-138, 1975.

Whalen, R. E. and B. B. Gorzalka. The effects of progesterone and its metabolites on the induction of sexual receptivity in rats. Hormones Behav. 3: 221-226, 1972.

27. Zucker, I. Facilitatory and inhibitory effects of progesterone on sexual responses of spayed guinea pigs. J. comp. physiol. Psychol. 62: 376-381, 1966.

28. Zucker, I. Actions of progesterone in the control of sexual receptivity of the spayed female rat.J. comp physiol. Psychol. 63: $313-316,1967$.

29. Zucker, I. Progesterone in the experimental control of the behavioral sex cycle in the female rat. J. Endocr. 38: 269-277, 1967.

30. Zucker, I. and R. G. Goy. Sexual receptivity in the guinea pig: Inhibitory and facilitatory actions of progesterone and related compounds. J. comp. physiol. Psychol. 64: 378-383, 1967. 